\title{
Megasporogenesis, Microsporogenesis, and Development of Female and Male Gametophytes of Ziziphus jujuba Mill. 'Zhongqiusucui'
}

\author{
Fengxia Shao, Sen Wang, Juan Chen, and Rongyan Hong \\ Key Laboratory of Cultivation and Protection for Non-Wood Forest Trees, \\ Ministry of Education, Central South University of Forestry and Technology, \\ Changsha 410004, China; Key Lab of Non-wood Forest Products of State \\ Forestry Administration, Central South University of Forestry and \\ Technology, Changsha, Hunan, 410004, China; and Engineering and \\ Technology Research Institute of Jujube Industry in Southern China, \\ Central South University of Forestry and Technology, Changsha, Hunan, \\ 410004, China
}

Additional index words. Ziziphus jujuba Mill. Zhongqiusucui, mega-and microsporogenesis, male gametogenesis, female gametogenesis

\begin{abstract}
To investigate whether reproductive disorders exist in the sexual reproduction of Ziziphus jujuba Mill. 'Zhongqiusucui' and to understand the reproductive biology of 'Zhongqiusucui' and genetic improvements in jujube trees, we used 'Zhongqiusucui' flowers at different developmental stages as materials and conducted field and microscopic observations on the developmental pattern of mega- and microsporogenesis, as well as on the development of male and female gametophytes. The results show the following. 1) From the inflorescence development stage to flowering, the grade 0 bud on the inflorescence exhibited an increase in horizontal diameter, longitudinal diameter, peduncle length, and bud weight, but the rates of increase were different. From day 1 to day 5 after the inflorescence had developed, floral buds mostly grew horizontally. Day 5 was the floral bud flattening stage. From day 6 to day 8 after the inflorescence had developed, floral buds mostly grew longitudinally, and day 8 was the floral bud enlarging stage. 2) The stamens of 'Zhongqiusucui' had five anthers, and there were four locules per anther. The anther wall consisted of epidermis, endothecium, one- to two-layered middle layer, and a secretory-type tapetum. In addition, the development of the anther wall belonged to the basic type. The cytokinesis of the microsporocytes was synchronous, the tetrads mostly arranged as a tetrahedron, and the mature pollen had three germ pores, three grooves, and was bicellular pollen. During meiosis, the microsporocytes in each locule were at the same phase and therefore exhibited synchrony. Among the different anthers in the same floral bud, as well as the four locules in the same anther, the microsporocytes had asynchronous meiosis. 3) The pistils in the 'Zhongqiusucui' had two ovaries, two anatropous ovules, inner and outer integument, crassinucellate tetrads formed by the meiosis of megasporocytes aligned linearly along the nucellus, megaspore at the chalazal end that developed into the functional megaspore, which underwent mitotic division three times and developed into the mature embryo sac containing seven cells and eight nuclei, and embryo sac development of the Polygonum type. 4) The external morphology of the 'Zhongqiusucui' floral buds correlated with the internal developmental stage of the male and female gametophyte. Therefore, the internal developmental progress of the stamen and pistil can be determined by the external morphological characteristics of the floral buds.
\end{abstract}

Ziziphus jujuba Mill. 'Zhongqiusucui' (Ziziphus jujuba Mill.) is a new cultivar bred in Hunan Province, and it has the characteristics of a large fruit size and high sweetness. 'Zhongqiusucui' has a cylindrical-shaped fruit, thin skin, thick flesh, small pit, an average fruit weight of $13.8 \mathrm{~g}$, and an edible rate up to $97.1 \%$. The fruit is juicy and has white-colored flesh, a fine and crispy texture, and light fragrance, and its soluble solid content is $31.76 \%$, its total sugar content is $29.41 \%$, and its flavor is balanced in sweetness (Wang et al., 2009). Compared with other major cultivars, such as Dongzao, Lizao, and Pingguodongzao in northern China, as well as Jidanzao in the Yangtze River Valley, 'Zhongqiusucui' is mostly cultivated in southern China, which has unique geographic and climate conditions. This fruit has many advantages, such as a high yield, high sugar content, rich flavor, good texture, strong adaptability, and easy storability and transportability, and it has become the main cultivar in southern China for fresh consumption. However, in fruit production, 'Zhongqiusucui' also faces dis- advantages, such as severe abscission of flowers and fruits and easy fruit cracking. To improve fruit quality and fruit setting rate, it is necessary to carry out crossbreeding and germplasm innovation to combine the advantages of other jujube cultivars. However, crossbreeding also introduces certain issues, such as seed abortion and nonsetting fruit. Research to identify the causes of seed abortions is urgently required because solving this challenge is critical for breeding new cultivars and improving fruit production.

Any disruption of the pollen, embryo sac, embryo, and endosperm developmental process would result in seed abortion (Liang et al., 2005). This study investigated the processes of microsporogenesis and megasporogenesis and the development of male and female gametophytes. Our goal was to elucidate whether reproductive disorders exist in the sexual reproduction of 'Zhongqiusucui' and to provide a foundation for carrying out crossbreeding.

\section{Materials and Methods}

\section{Experimental site}

The experimental site was the jujube tree experimental base at Central South University of Forestry and Technology (Qidong County, Hunan Province, China), which is located at lat. $26.78^{\circ} \mathrm{N}$, long. $112.12^{\circ} \mathrm{E}$.

\section{Experimental cultivars}

Five-year old Z. jujuba Mill. 'Zhongqiusucui' trees that had been transplanted once were used in this study. We selected trees with good growth and developmental conditions, relatively uniform tree vigor, and no disease or pests.

\section{Experimental methods}

Sample observation and treatment. At the inflorescence developing stage, a large number of inflorescences on the top of different bearing shoots at the middle of the canopy were selected and marked. Five inflorescences were selected and followed from the beginning of the inflorescence developing stage. At 10 AM every day, pictures of grade 0 buds were taken using a Canon macro camera (EOS 5D Mark IV, EF $100 \mathrm{~mm} \mathrm{f} / 2.8 \mathrm{~L}$ IS USM; Canon, Tokyo, Japan) to record their growth and development. Twenty inflorescences were selected, and the grade 0 buds on the inflorescence were measured every day using a Vernier caliper to record the horizontal diameter (the width of flower bud), longitudinal dimeter (the vertical height from the base to the top of flower bud), and peduncle length. From the marked inflorescences, 30 flower buds were collected every day. Fifteen buds were immediately immersed in formalin-acetic acid-alcohol fixative (50\% ethanol:acetic acid:formaldehyde $=$ 90:5:5). Samples were placed in a vacuum to pull air out of the tissue and then placed in a $4{ }^{\circ} \mathrm{C}$ refrigerator for storage. For the other 15 floral buds, the weights of single 
buds were measured using an electrical analytical balance.

Microscopic observations. The routine paraffin section method (Li, 2009) was used to generate sections of $8-\mu \mathrm{m}$ thickness. Sections were stained using the modified Ehrlich's hematoxylin staining method (Xu et al., 2008) and mounted with neutral gum. A DMi8 inverted microscope (Leica, Wetzlar, Germany) was used to observe the processes of microsporogenesis and megasporogenesis as well as the development of male and female gametophytes. Photographs were taken to record the observations.

\section{Results and Analysis}

\section{Observations of jujube morphology during the floral bud stage}

The flowering period of single jujube flowers began from the appearance of floral buds and lasted until the enlargement of ovaries. Qu et al. (1989) divided the jujube flowering process into three main stages: the floral bud stage, flowering stage, and young fruit stage. Three stages are included within the floral bud stage: floral bud appearance, inflorescence development, and floral bud yellowing stage. The appearance of the floral bud stage occurred when the floral buds first appeared at leaf axils. The inflorescence developing stage occurred when the axillary buds first appeared below one side of the grade 0 buds on the inflorescence. The floral bud yellowing stage occurred when floral buds turned yellowish green or yellow from green. Based on previous observations and preliminary experiments, we selected floral buds that were at the inflorescence developing stage, as well as those a few days past the inflorescence developing stage, as observation objects for this study.

From day 1 at the inflorescence developing stage, the marked grade 0 buds on the inflorescence were observed and photographed (Fig. 1). On the day when the inflorescence developed (Fig. 1A), the grade 0 buds were relatively small and half-covered

Received for publication 5 June 2019. Accepted for publication 8 July 2019.

The key research and development project of Hunan Province "Research and Demonstration of Technology for Improving Quality, Increasing Yield and High Value Utilization of Woody Grain Tree Species in Hunan Province (2018NK2043)," the Key Research and Development Project of Hunan Province "Quality Innovation and Key Technologies of Main Nonwood Forest Species in Hunan Province (2016NK2147)," and the Postgraduate Science and Technology Innovation Fund Project of Central South University of Forestry Science and Technology "Research on Embryo Abortion Mechanism of Fresh-eating Jujube in South China (CX2016A02)" provided financial support for the conduct of the research and preparation of the article. Thanks are due to Juan Chen and Rongyan Hong for assistance with the experiments, and to Professor Sen Wang for valuable discussion.

S.W. is the corresponding author. E-mail: csuftwangsen@163.com. by bracts. Five sepals were not obvious. On one side of the grade 0 buds, lateral young grade 1 buds could be observed. As the grade 0 buds gradually enlarged, their colors also changed. From day 1 to day 3 , the grade 0 buds were bright green-colored (Fig. 1A-C). On day 4, the color of the grade 0 buds changed to green (Fig. 1D), which slightly darkened on day 5 (Fig. 1E). The green color was maintained from this point to day 9, when the grade 0 buds appeared to be yellowish green (Fig. 1I). On day 12, the yellow color started to become more obvious (Fig. 1L). On day 13, the floral buds were enlarged to their maximum size and were yellow-colored (Fig. 1M). A small fraction of the grade 0 buds began to open and blossom (Fig. 1N). From this point, all observed floral buds blossomed.

To further study the growth and developmental pattern of the grade 0 buds on different days after the first appearance of the inflorescence, we measured and calculated their horizontal diameter. The results are shown in Figs. 2 and 3.

As shown in Fig. 2, at different times at the inflorescence developing stage, the grade 0 buds exhibited an increase in horizontal diameter, longitudinal diameter, peduncle length, and bud weight. The horizontal and longitudinal diameters of the floral buds did not exhibit any significant difference on day 1 at the inflorescence developing stage, whereas they did show some differences in the following days. From day 1 to day 5 at the inflorescence developing stage, the bud horizontal diameter increased faster than the longitudinal diameter, and this different rate of increase could also be confirmed by the ratio of these two values. As shown in Fig. 3, the ratio of longitudinal diameter/horizontal diameter exhibited a decrease from day 1 to day 5 , indicating that during this period, the floral buds were mostly elongated horizontally, and on day 5 , the floral buds had a rather flattened shape. In previous studies on jujube flower development, this stage was called the flat bud stage (Zeng, 1959). From day 5 to day 6, the ratio of the increase in horizontal and longitudinal diameter stayed the same, followed by an increase in the ratio until reaching its peak on day 8 . This finding suggests that from day 6 to day 8 , the floral buds grew mostly in the longitudinal direction. At this point, the floral bud longitudinal diameter was larger than it was previously, and the shapes of the buds were rounder. This stage was referred to as the floral bud enlarging stage (Zeng, 1959). From day 8 onward, the ratio of longitudinal and horizontal diameters exhibited a small degree of fluctuation. Figure 2 also shows that the length of the peduncle had a rate of increase similar to those of the longitudinal and horizontal diameter from day 1 to day 6 , which was followed by a rapid increase, and the peduncle length eventually exceeded the bud longitudinal diameter on day 9 . From day 7 to day 13 , the length of the peduncle continued a gradual and steady increase but stayed shorter than the horizontal diameter of the bud. The floral bud weight began to rapidly increase from day 4, at a rate faster than all the other parameters. This result may be because on day 4 at the inflorescence developing stage, individual parts of the floral buds began to fully differentiate, and the accumulation of nutrients was faster.

\section{Microsporogenesis and male gametophyte development}

Anther wall development. The anther of 'Zhongqiusucui' contained four locules. During the early stages of development, the stamen primordia formed anther primordia (Fig. 4A), and anther differentiation had not yet begun. A layer of epidermis was on the outside of the anther primordia. Underneath the epidermis, a group of actively dividing cells was observed. Because cells in the four corners of the anther were dividing more rapidly, the anther developed into a fourangled shape. Under the epidermis of each angle, archesporial cells were differentiated. Many archesporial cells with dense cytoplasm were seen on the cross sections (Fig. 4B). After the formation of archesporial cells, they divided periclinally and further differentiated into primary parietal cells and primary sporogenous cells (Fig. 4C and D). The primary parietal cells continued to divide periclinally and vertically and produced a series of cells arranged in concentric circles of usually three to five layers. Together with the outmost epidermis, they formed the anther wall (Fig. 4E and F). The anther wall of 'Zhongqiusucui' fully differentiated at the microspore mother cell stage (Fig. 4G), and it contained four layers: the epidermis, endothecium, middle layer, and tapetum (from the outermost to the innermost layers). The middle layer was a single layer of cells. During the meiosis of the microsporocyte, cells in the middle layer already began to differentiate and showed decreased storage substances, and the cells flattened and gradually degenerated and were absorbed (Fig. 4H-O). The middle layer was absent in the mature anther of 'Zhongqiusucui' (Fig. 4U). During the development of the anther wall, the tapetum was rich in nutrients and provided sufficient material for the development of microspores. Thus, the tapetum was the most conspicuous during the entire anther developmental process. These findings are similar to the results of Chunyan He (2009). During meiosis I prophase of the microspore mother cell, the tapetum began to thicken radially (Fig. $4 \mathrm{H}$ ). When meiosis began, the tapetum started to degrade gradually. At the stage of microspore tetrads, the tapetum began to disintegrate in situ (Fig. 4P) and continued degrading during the mononucleus stage. This led to the separation of the tapetum from the middle layer of the anther wall (Fig. 4Q and R). When the pollen of 'Zhongqiusucui' was maturing, the tapetum started to dissolve and degenerate and eventually disappeared. As a result, the anther wall of 'Zhongqiusucui', originally with four layers, contained only two layers at this point: the endothecium and epidermis (Fig. 4U). The anther wall development in 'Zhongqiusucui' belonged to 

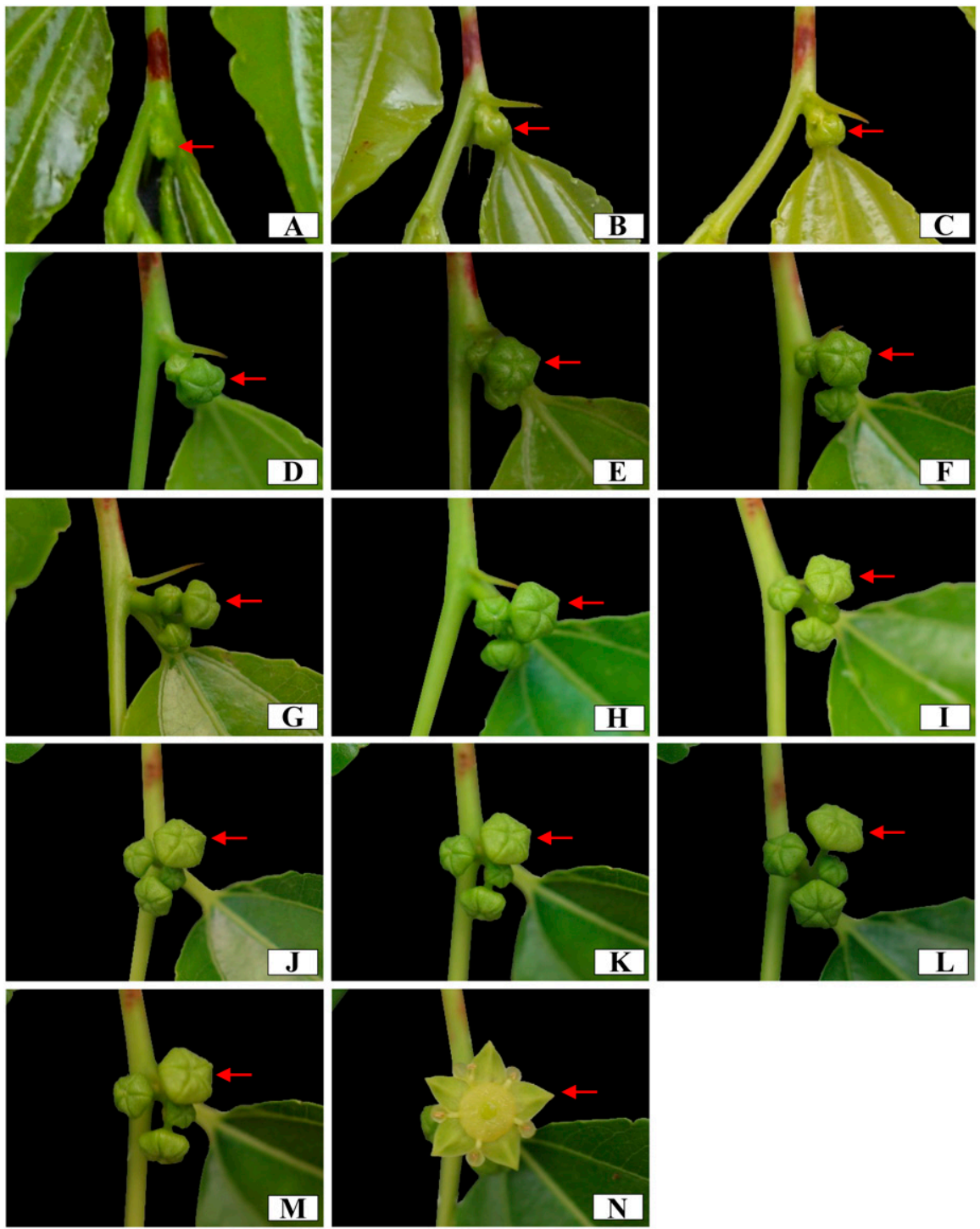

Fig. 1. Morphological development characteristics of flower buds at the inflorescence developing stage. (A) The grade 0 bud on the inflorescence in day 1 (arrow). (B) The grade 0 bud on the inflorescence on day 2 (arrow). (C) The grade 0 bud on the inflorescence in day 3 (arrow). (D) The grade 0 bud on the inflorescence on day 4 (arrow). (E) The grade 0 bud on the inflorescence on day 5 (arrow). (F) The grade 0 bud on the inflorescence on day 6 (arrow). (G) The grade 0 bud on the inflorescence on day 7 (arrow). (H) The grade 0 bud on the inflorescence on day 8 (arrow). (I) The grade 0 bud on the inflorescence on day 6 (arrow). (J) The grade 0 bud on the inflorescence on day 10 (arrow). (K) The grade 0 bud on the inflorescence on day 11 (arrow). (L) The grade 0 bud on the inflorescence on day 12 (arrow). (M) The grade 0 bud on the inflorescence on day 13 (arrow). (N) The grade 0 opening flower on the inflorescence on day 13 (arrow).

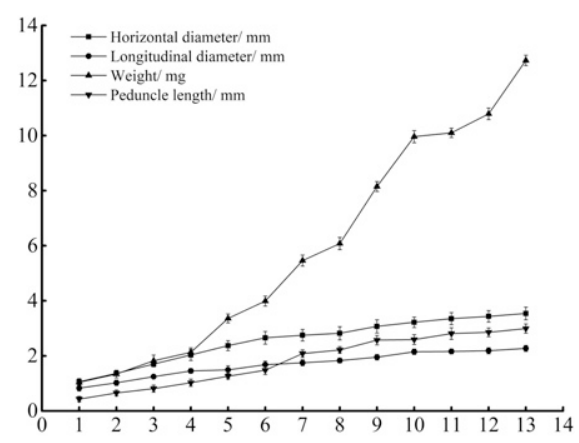

Days at the inflorescence developing stage/ $\mathrm{d}$

Fig. 2. Changes of Zizyphus jujuba Mill. 'Zhongqiusucui' bud size with time. the basic type (Davis, 1966). Based on the morphological differences of tapetum during its later stages of development, Davis (1966) divided tapetum into two types: the plasmodial type and secretory type. The degeneration and dissolution of the tapetum in 'Zhongqiusucui' occurred at its original location. therefore, its nucleus and cytoplasm also dissolved at the original location without changing. Thus, the tapetum in 'Zhongqiusucui' was the secretory type, which is consistent with that in 'Dongzao' (Huang et al., 2017) and 'Jinsi No.4' (Zou et al., 2013).

Microsporogenesis. The archesporial cell divided and formed the primary sporogenous cell, which then generated secondary spo- rogenous cells through multiple rounds of mitosis. The secondary sporogenous cell continued to develop into microspore mother cells that had characteristics of a large cell volume, large nucleus, and dense cytoplasm (Fig. 4G). The microsporocyte sequentially went through meiosis I prophase (Fig. 4H), metaphase (Fig. 4I), anaphase (Fig. 4J), and telophase (Fig. 4K) and then completed meiosis I and formed two daughter nuclei. Without forming a cell wall between the two daughter nuclei, the cells entered meiosis II and went through prophase (Fig. 4L), metaphase (Fig. 4M), anaphase (Fig. 4N), and telophase (Fig. 4O), and then tetrads were formed (Fig. 4P). The tetrads were mainly tetrahedrally shaped, and a few were symmetrical. Newly formed four haploid microspores were surrounded by a shared callose wall, and each microspore lacked its own cell wall. When tetrads subsequently began to degenerate, the callose wall gradually dissolved. In tetrad microspores, the cell wall was formed and thickened, thereby leading to the development of free microspores (Fig. 4Q). The materials of the pollen wall were provided only by the cytoplasm of microspores during the tetrad stage. after the release of microspores, the wall materials came from not only the microspores themselves but also the tapetal cells ( $\mathrm{Hu}, 2005)$. The meiosis process of microspores showed that cytokinesis was simultaneous.

The microsporocytes in each locule were at the same stage during meiosis and exhibited synchrony. In different anthers on the same floral bud as well as the four locules on the same anther, the meiosis of the microspore mother cells was asynchronous and showed a difference of two to five phases. As shown in Fig. 5A-C, in the same floral bud, one anther was at the prophase of meiosis I (Fig. 5C), while another anther was at the tetrad stage (Fig. 5B). In Fig. 5D, in the same anther, one pollen sac was at the anaphase of meiosis I while another one was at the telophase of meiosis II.

Male gametophyte development. The development of male gametophytes in 'Zhongqiusucui' began with microspores. Because the callose in the tetrad of microspores degraded, dissolved, and disappeared, the microspores were gradually released from the tetrad and formed haploid microspores. The formation of haploid microspores marked the beginning of microspores entering the male gametophyte developmental stage. When microspores were first released from the tetrad, they had thin cell walls, dense cytoplasm, nucleus in the middle of the cell, and an overall small cell volume (Fig. 4Q). Microspores absorbed nutrients from their surrounding tissues and organs during their development, which promoted the further development of microspores into haploid microspores with an increased cell volume and enlarged nucleus (Fig. 4R). During the development of microspores, three germ pores formed on the surface on the microspores, and they functioned as channels for nutrients provided by the tapetum to enter 
microspores. As microspores continued absorbing water and nutrients from the tapetum, their cell volume increased, with a large

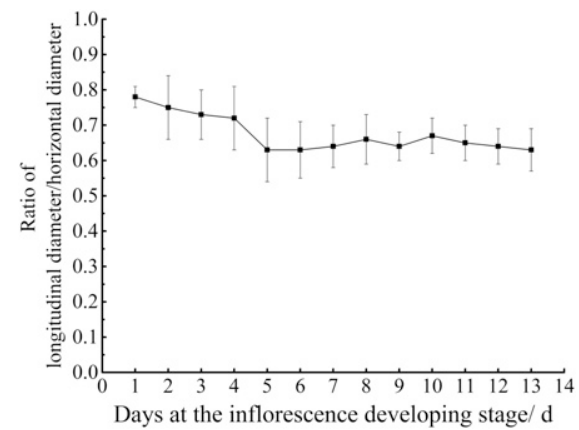

Fig. 3. Variation of the ratio of longitudinal and transverse diameters of jujube buds with time. vacuole forming in the center of the cell. The nuclei gradually moved to the cell periphery, and microspores entered the uninucleate microspore stage (Fig. 4S). After this stage, the haploid cell underwent an asymmetric mitotic division into diploid cells, with the larger cell as the vegetative cell and the smaller cell as the generative cell (Fig. 4T). As the pollen grains increased in volume, the cell wall between the generative cell and the vegetative cell disappeared. The generative cell gradually migrated into the cytoplasm of the vegetative cell and formed bicellular pollen, where the generative cell was round shaped with a relatively large nucleus. The bicellular pollen continued to develop into a mature pollen grain that had a triangle shape, three germ pores, and three grooves (Fig. 4U). The mature pollen grain is bicellular. During the development of mi-

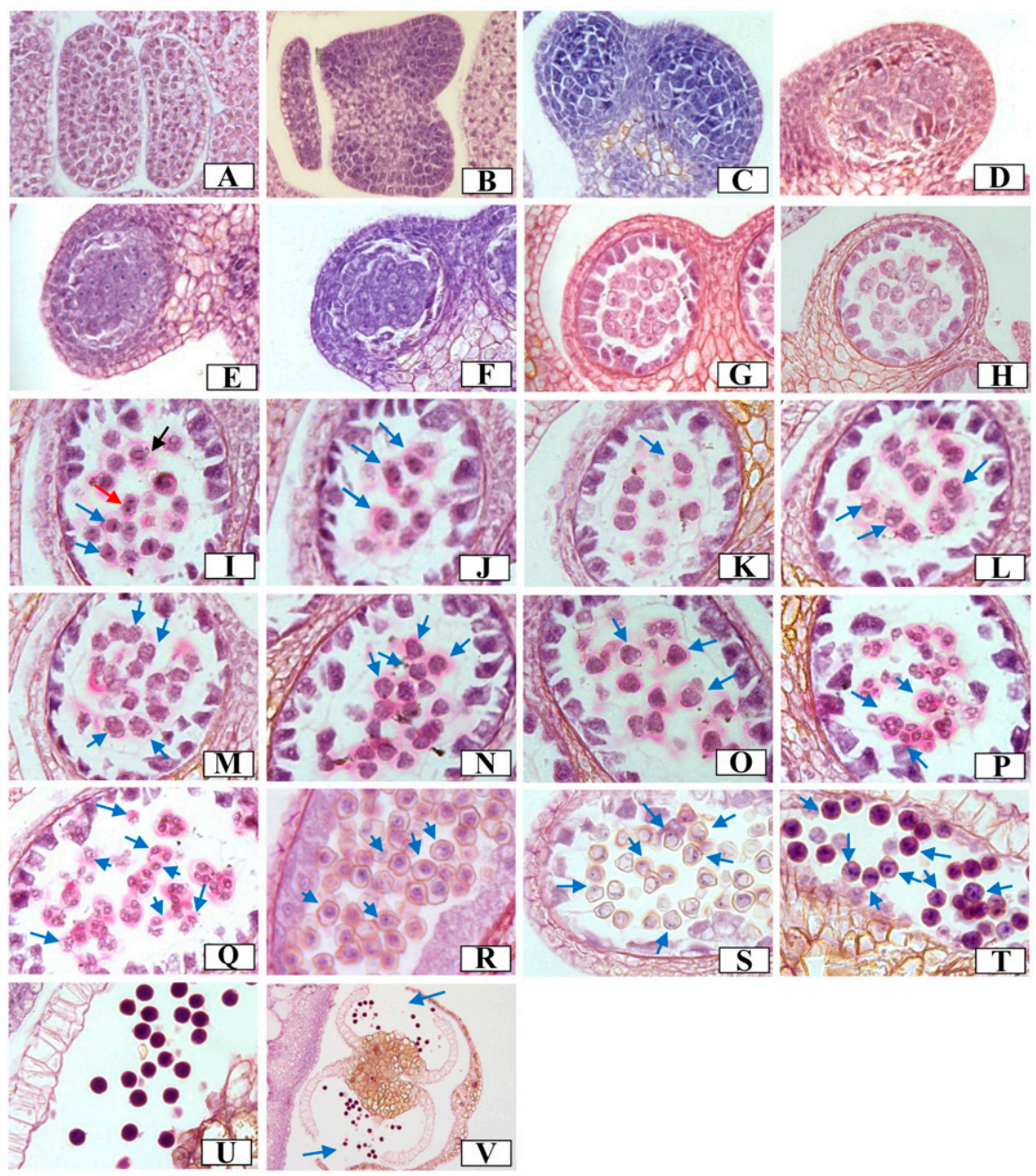

Fig. 4. Microsporogenesis and male gametophyte development. (A) Anther primordium. (B) Archesporial cell. (C) Periclinal division of archesporial cell. (D) Differentiation of anther inner wall and sporogenous cell. (E) Differentiation of anther inner wall and primary sporogenous cell. (F) Early stage tapetum and secondary sporogenous cell. (G) Completion of anther wall development and microsporocytes. (H) Meiosis I prophase. (I) Meiosis I metaphase (blue arrow), early anaphase (black arrow), and late anaphase (red arrow). (J) Meiosis I late anaphase (blue arrow). (K) Meiosis I telophase (blue arrow). (L) Meiosis II prophase (blue arrow). (M) Meiosis II metaphase (blue arrow). (N) Meiosis II anaphase (blue arrow). (O) Meiosis II telophase (blue arrow). (P) Tetrad phase (blue arrow). (Q) Microspores released from tetrads (blue arrow). (R) Early uninucleate microspore stage (blue arrow). (S) Late uninucleate microspore stage (blue arrow). (T) Binucleate stage (blue arrow). (U) Mature pollen grain, dense cytoplasm, with darker staining. (V) Mature pollen grain stage, cracked between two locules on the anther, pollen grain ready to disperse. crospores, we observed abnormal microspore development. Aborted microspores shrunk in size and contained no nucleus (Fig. 5E). Pollen was absent in a few pollen sacs and presented degenerated and disappeared tapetum layers (Fig. 5F). We also observed an abnormal pollen sac, as shown in Fig. 5G. One anther had three locules at the uninucleate microspore stage, and one pollen sac presented no microspores and was filled with an unidentified substance. In addition, the same situation as mentioned previously was observed in the pollen sac of the entire anther (Fig. 5H). These observations of microspore development had not been previously reported in other jujube cultivars. We speculated that this development pattern may be due to abnormalities in the tapetum. however, detailed causes have not been elucidated and further studies are required.

\section{Megasporogenesis and female gametophyte development}

Megasporogenesis. The timing of megasporogenesis in 'Zhongqiusucui' was obviously later than that of microsporogenesis. We did not observe ovule primordia in the ovary until microspores entered meiosis. The ovule primordia initiated from the placenta of the ovary wall (Fig. 6A). The apical cells in the ovule primordia further differentiated and formed the nucellus (Fig. 6B and C). Under the nucellus epidermis, one cell differentiated into the archesporial cell (Fig. 6D). This archesporial cell was different from other cells in the nucellus because of its large volume, dense cytoplasm, and inclusion of an obvious nucleus. Next, the cells in the nucellus tissue differentiated into inner and outer integuments. The outer integument developed more quickly than the inner integument. therefore, it gradually surrounded the nucellus and the inner integument. In later stages, one side of the outer integument accelerated its development, which caused the ovule to gradually bend and eventually develop into an anatropous ovule (Fig. 6E and F). There were two ovules in most of ovaries, one ovule in each chamber of the ovary. Meanwhile, we observed three ovules in a few numbers of ovaries (Fig. 7A and B)

The archesporial cell in the nucellus divided periclinally and produced a parietal cell and a sporogenous cell (Fig. 6E), with the latter functioning as the megaspore mother cell. The megaspore mother cell underwent meiosis I and formed dyads (Fig. 6E), which then continued to divide and formed four megaspores arranged linearly along the nucellus (Fig. 6F). Three megaspores close to the micropyle gradually degenerated. Only one megaspore at the chalazal end became the functional megaspore. Generally, based on the development of the archesporial cell, ovules can be divided into two forms: the tenuinucellar and crassinucellar forms. The tenuinucellar ovule refers to the megaspore mother cell that is directly underneath the nucellus epidermis without any parietal cells. In the crassinucellar ovule, the megaspore mother cell was separated from the 


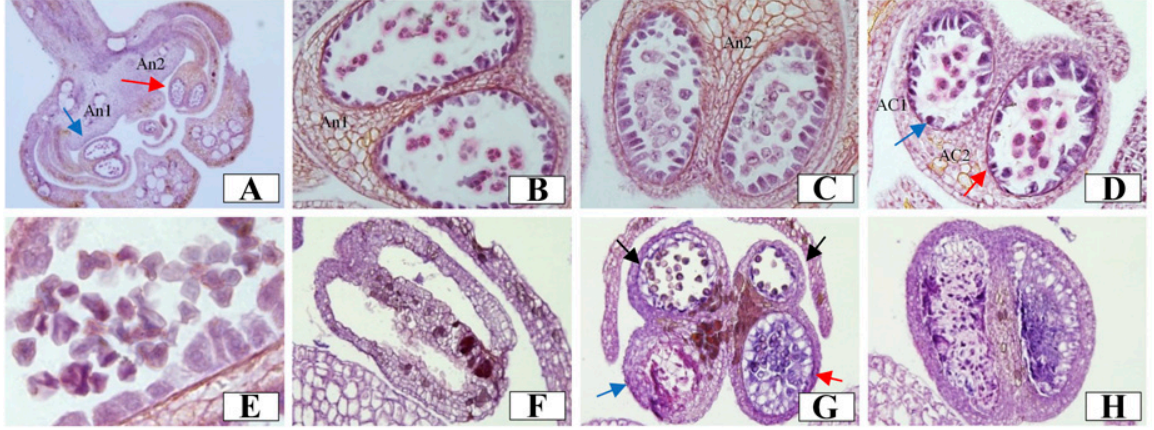

Fig. 5. Simultaneous phenomenon and abnormal microspore development. An = anther; $\mathrm{AC}=$ anther chamber. (A) Two anther in one bud. (B) Anther 1 of the bud in Fig. 1, staying tetrad phase. (C) Anther 2 of the bud in Fig. 1, staying meiosis I prophase. (D) In the same anther, a pollen sac in meiosis I late anaphase (blue arrow), and another at the meiosis II telophase (red arrow). (E) Abnormally developed pollen. (F) Empty pollen sac. (G) In the same anther, two 2 chambers developed normally, and in early uninucleate microspore stage (black arrow). The other two did not develop normally. There were no microspores and filled with some unknown plasmids in one of the other two chambers (blue arrow). Microspores in the other one were abnormal development and surrounded by unknown plastid closely (red arrow). (H) Abnormal anther filled with unknown plastids.
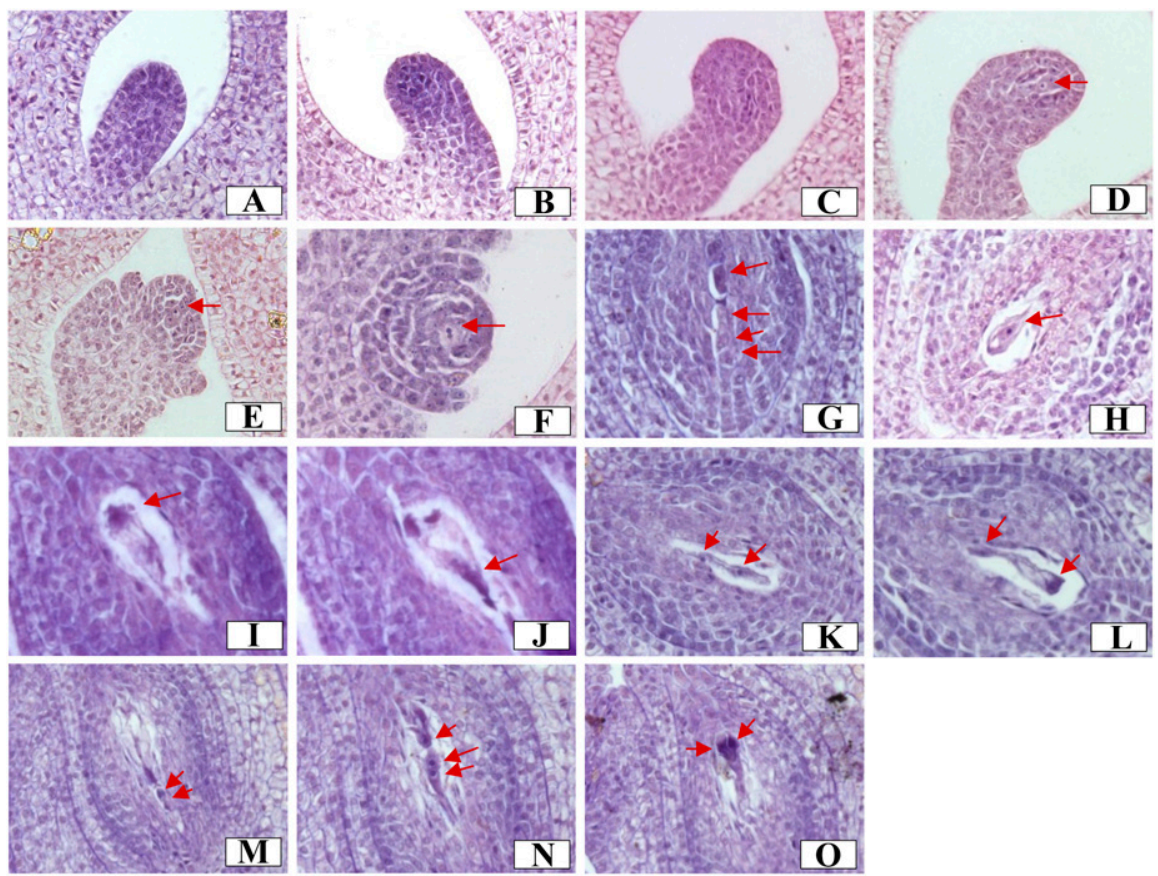

Fig. 6. Megasporogenesis and female gametophyte development. (A) Ovule primordium. (B) Ovule primordium differentiation. (C) Ovule primordium differentiation. (D) Archesporial cell (arrow). (E) Peripheral cells and sporogenic cells (arrow). (F) Dyad period (arrow). (G) Tetrad period. The three megaspores near the micropyle are degenerating (arrows). (H) Mononuclear embryo (the arrow indicates a functional megaspore). (I, J) Two-nucleate embryo sac (arrow). (I) The chalazal end nucleus (arrow). (J) The nucellar end nucleus on all other surfaces of the same embryo sac (arrow). (K, L) Period of tetranuclear embryo sac: two sections of the same embryo sac. (K) The binucleus near the chalaza and micropyle, respectively (arrow), and $(\mathbf{L})$ the other binucleus near the chalaza and micropyle, respectively (arrow). (M, L, O) Mature embryo sac period: Three aspects of the same embryo sac, respectively. (M) Antipodal cells near the chalaza (arrow). (N) The oocyte near the micropyle (arrow), the central cell containing two polar nuclei (arrow), and antipodal cells (arrow), and one antipodal cell near the chalaza (arrow). (O) Two synergid cells near the micropyle (arrow).

nucellus epidermis by one or a few layers of parietal cells (Hu, 2005). The megaspore mother cell in 'Zhongqiusucui' was separated from the nucellus epidermis by multiple layers of cells. therefore, its ovule belonged to the crassinucellar form.

Female gametophyte development. The functional megaspore further developed into a uninucleate embryo sac with a large nucleus and an increased volume (Fig. 6H). The uninucleate embryo sac underwent mitotic division once and formed two nuclei, which were pushed by the vacuole and migrated to two poles to form the two-nucleate embryo sac (Fig. 6I and J). Another mitotic division resulted in a four-nucleate embryo sac
(Fig. 6K and L). The four nuclei on both sides of the embryo sac underwent another mitotic division and formed the eightnucleate embryo sac, with four nuclei at the micropyle end and the other four nuclei at the chalazal end. One nucleus from the micropyle end migrated downward, one nucleus from the chalazal end migrated upward, and they together formed two parallel nuclei at the center of the ovule. The stage of the eight-nucleate embryo sac lasted only briefly, and cell differentiation began quickly after. As the eight-nucleate embryo sac volume increased and cellularization completed, the embryo sac entered its mature stage (Fig. 6M-O). In the mature embryo sac, the egg cell and two synergid cells were at the micropyle end, and three antipodal cells were at the chalazal end in the nucellus tissue. Two polar nuclei were in the center of the embryo sac. The mature embryo sac laid on top of the nucellus. A stalk-like structure that served as a pedestal formed underneath the embryo sac in the nucellus tissue of the ovule. From the developmental pattern of the embryo sac in 'Zhongqiusucui', we concluded that the structure of its embryo sac was the Polygonum type.

We observed some abnormally developed embryo sacs during female gametophyte development. Some embryo sacs had no complete eight nuclei, or scattered with approximately two to four irregular nuclei, which were often degenerated, with the absence of single or multiple antipodal cells, egg cells, and helper cells (Fig. 7C-F). As shown in Fig. $7 \mathrm{C}$, there were only polar nucleus and egg cells in the embryo sac, and there were no antipodal cells and helper cells in the adjacent sections of the embryo sac. The embryo sac lacked antipodal cells, helper cells, egg cells, and another polar nucleus in Fig. 7D. In Fig. 7E and F, the embryo sac was scattered with approximately two to three irregular nuclei, with the absence of antipodal cells, helper cells, egg cells, and polar nuclei. Antipodal cells were not observed in most abnormally developed embryo sacs. Antipodal cells generally degenerate at the mature embryo sac stage or shortly after fertilization (Hu, 2005). Therefore, in this study, antipodal cells degenerated earlier. There were only traces of degenerated cells (Fig. 7G and $\mathrm{H}$ ), or only a small number of inclusions, or completely empty (Fig. 7I and J) in some other embryo sacs. Because of the abnormal development of the embryo sac, the ovule loses its vitality. These embryo sacs were considered abnormal. As a result, although somewhat full ovules in the ovary locules were visible, under the microscope, these ovules were empty sacs with only the integument.

Abnormal ovules also were observed. Some ovules were deformed early in development (Fig. 7K and L) or aborted (Fig. 7M and $\mathrm{N}$ ). There were also some other ovules with hypertrophic nucellus protruding out of the integument and containing abnormally developed embryo sacs (Fig. 7O). 

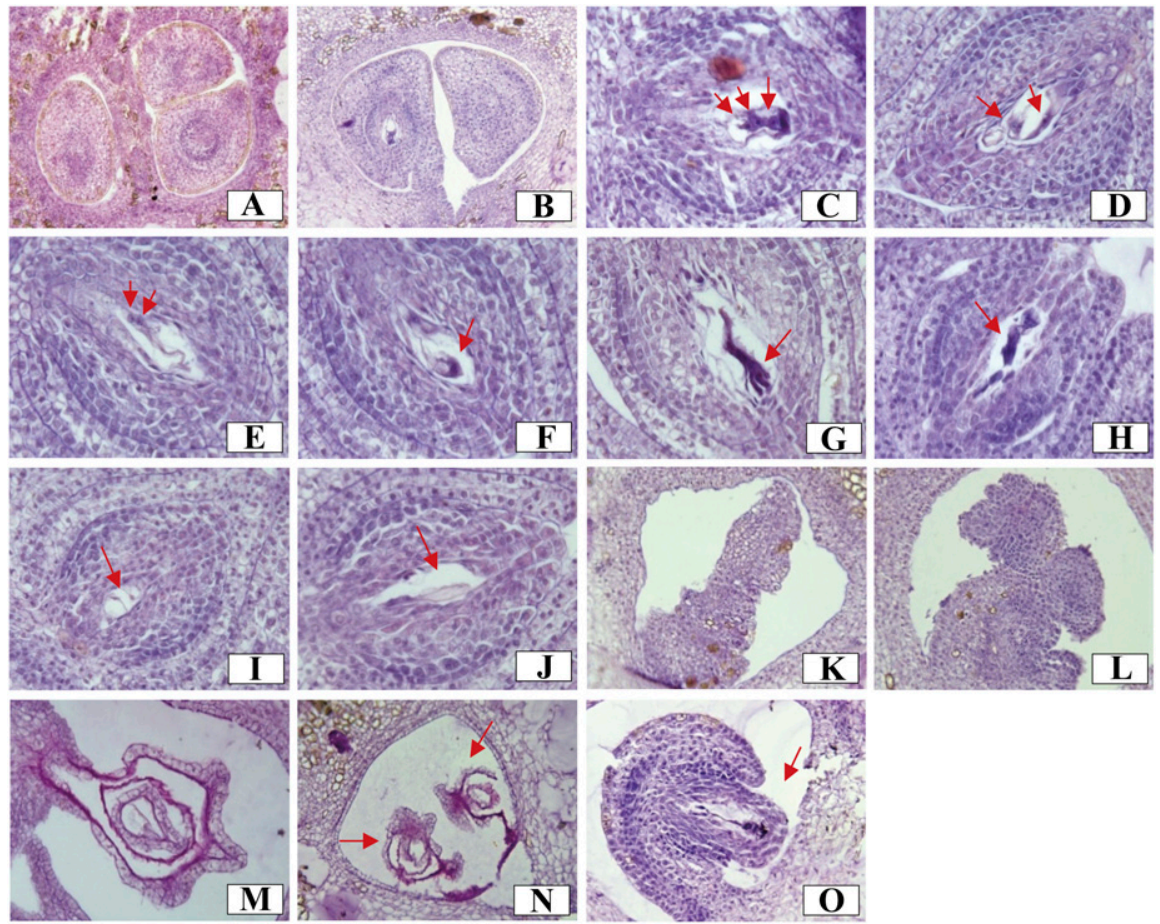

Fig. 7. Three ovules in one ovary and abnormally developed embryo sacs. (A) Cross section of three ovules in one ovary. (B) Longitudinal section of two ovules in one chamber of ovary. (C) Abnormal embryo sac with only polar nucleus and egg cells (arrows). (D) Abnormal embryo sac with only polar nucleus and one antipodal cell (arrows). (E, F) Abnormal embryo sac with two to three scattered irregular nuclei (arrows), missing antipodal cells, helper cells, egg cells and polar nuclei. (G, H) Abnormal embryo sac containing only the vestigial traces of a group of degenerated cells (arrow). (I) Abnormal embryo sac with a small number of inclusions (arrow). (J) Abnormal embryo empty sac (arrow). (K, L) Deformed ovule. (M) Degenerated abortive ovule. (N) Two degenerated ovules in one ovary (arrows). (O) Hypertrophic nucellus containing an abnormally developed embryo sac (arrow).

\section{Correlation between the size and morphology of floral buds with stages during male and female gametophyte development}

As shown in Table 1, in 'Zhongqiusucui', the development of the stamen and pistil was asynchronous. However, as a hermaphroditic plant, the developmental progress of both male and female gametophytes in the same floral bud could be observed simultaneously. The stamens developed earlier than the pistils throughout the entire development process of male and female gametophytes. When microspores began meiosis, the ovule primordia first started to appear in the pistil. When the haploid microspores were at the center or at the periphery, we first observed the megaspore mother cell, which was followed by the accelerated development of the embryo sac. When pollen matured, the embryo sac had not reached its maturity. Until 2 to $3 \mathrm{~d}$ after anther burst, the embryo sac became mature. We combined and analyzed the observations of floral bud morphology from the inflorescence developing stage to flowering stage with the development of male and female gametophytes within floral buds. We found that the floral bud morphology of 'Zhongqiusucui' was correlated with the development of male and female gametophytes. In other words, we could speculate on the developmental condition of male and female gametophytes from the developmental age of floral buds, phenophase, bud size, and external morphology.

\section{Conclusions and Discussion}

From the inflorescence developing stage to flowering stage, the development of the floral bud stage was normal. The grade 0 floral buds in the inflorescence exhibited an increase in horizontal and longitudinal diameter, peduncle length, and bud weight, although the rate of increase of these parameters differed. From day 1 to day 5 of the inflorescence developing stage, the horizontal diameter of the floral buds increased at a higher rate than the longitudinal diameter. Floral buds mainly elongated horizontally. At day 5 from the development of inflorescence, the floral bud flattening stage was observed. From day 6 to day 8 at the inflorescence developing stage, floral buds grew mainly in the longitudinal direction. Day 8 marked the beginning of the floral bud enlarging stage. This finding is different from the observations by Qu et al. (1989), who stated that it took $\approx 20 \mathrm{~d}$ from the inflorescence developing stage to floral bud yellowing stage. One possible reason for this discrepancy could be related to the different jujube cultivars. In the study by $\mathrm{Qu}$ et al. (1989), experimental cultivars were Lingzao, Pozao, Mayazao, Huizao, Hupingzao No. 1 and No. 2, and Suanzao, which were different from the cultivar used in this study, that is, the 'Zhongqiusucui'. Another possible reason could be the different climates between southern and northern China. In southern China, the temperature is higher, and the daylight is longer, which shortens the time needed for floral bud development and flowering. The floral bud weight started to rapidly increase from day 4 at the inflorescence developing stage, with a rate of increase that was higher than that of the other three parameters. This finding may be because on day 4 , pistils began to develop and the differentiation of each part in the floral bud was becoming more complete. In addition, the accumulation of nutrients had also become more rapid. After the inflorescence developing stage, the color of the floral bud changed as follows: bright green - green - yellowish green - yellow. It took $\approx 12-13 \mathrm{~d}$ for the floral bud to develop from the inflorescence developing stage to the flowering stage.

The embryological characteristics of 'Zhongqiusucui' were consistent with those reported in 'Dongzao' (Huang et al., 2017), 'Jisi No. 4' (Zou et al., 2013), 'Changhongzao’ (Wang, 1983), 'Ningxiayuanzao’ (Tian and Ma, 1987), 'Huizao' (Zhang et al., 2004), and 'Suanzao' (Niu et al., 2011).The anther of 'Zhongqiusucui' had four locules, and the anther wall consisted of the epidermis, endothecium, one to two layers of middle layer, and tapetum. In addition, basic-type anther development and secretory-type tapetum development were observed. During the development of the anther wall, cells in the middle layer gradually degenerated or disappeared. The cytokinesis of microspores was simultaneous. The tetrads mainly had a tetrahedral arrangement, and a few had a symmetrical arrangement. Mature pollen grains were bicellular and presented three germ pores and three grooves. In the 'Zhongqiusucui' anther, the microsporocytes in each locule were at a similar phase during meiosis and presented synchronous characteristics. However, there was desynchronization occasionally. Among the five anthers within the same floral bud, meiosis of the microspore mother cells was asynchronous. This asynchrony was also true among four locules within the same anther. The difference could be two to five phases. A related study showed that before meiosis started, cells in the same layer of the anther wall, as well as adjacent cell layers, were connected by plasmodesmata $(\mathrm{Hu}$, 2005). The microspore mother cells within one pollen sac were connected by plasmodesmata, through which solutes and other factors could move from one microspore mother cell to another. This was considered the reason for the synchrony in meiosis (Heslop-Harrison, 1966; Whelan, 1974). When microspore mother cells underwent meiosis, the callose gradually deposited on the wall of the microspore mother cells while plasmodesmata connections were broken. The secreted callose existed between the primary cell wall and cytoplasm. Microspores formed by meiosis were surrounded by a shared callose wall, and they were also 


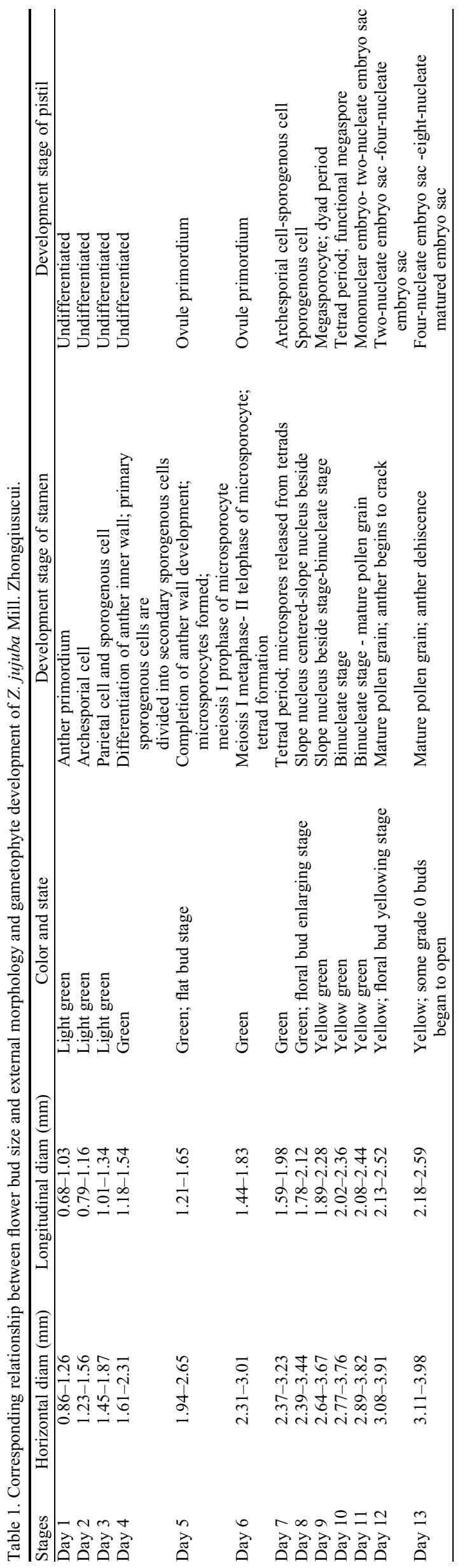

separated from each other by callose. Thus, microspore mother cells, tetrads, or microspores were separated or isolated, which led to a lack of synchrony after meiosis started. However, whether plasmodesmata were present among the pollen sacs and anthers was not clear, although the development was synchronous among different pollen sacs and anthers before meiosis. As a result, structural connections cannot sufficiently explain the reasons for this synchronous phenomenon. thus, further studies are required to investigate the causes. This asynchronous meiosis of microspore mother cells was also observed in other jujube cultivars, such as Dongzao (Huang et al., 2017) and 'Jinsi No. 4' jujube (Zou et al., 2013). Many studies have focused on the asynchronous phenomenon during the meiosis of microspore mother cells in other plant species (Meng et al., 2009). During microsporogenesis and male gametophyte development, we did not observe a large amount of aborted pollen, indicating that the pollen in 'Zhongqiusucui' was fertile, and aborted pollen could not explain embryo abortion in the jujube fruits. Abnormal development of the pollen, pollen sac, and empty pollen sac was occasionally observed, which might be related to the disappearance and degeneration of tapetum ( $\mathrm{He}$ and $\mathrm{Wu}$, 2013; Zhang et al., 2008, 2014). The specific reasons need to be further studied.

'Zhongqiusucui' has two-locular ovaries, two ovules in most of the ovaries (only a few ovaries contain three ovules), anatropous ovules, double integuments, and crassinucellar ovules. The megaspore mother cell underwent meiosis twice and formed tetrads that aligned linearly along the nucellus. Three megaspores near the micropyle end gradually degenerated, and one megaspore at the chalazal end became the functional megaspore. Seventy percent of the plants studied had a monosporic embryo sac, in which only one megaspore differentiated into the functional megaspore, whereas the other three megaspores did not differentiate or divide. In some species, a differently positioned megaspore differentiates into the functional megaspore, indicating the origin of the functional megaspore is regulated by a flexible developmental pathway. In plants such as Arabidopsis (Webbm and Gunning, 1990), callose deposition is closely correlated with the selection of a functional megaspore. The callose wall exists as a physical barrier, blocking the material and information communication between megaspores and their surrounding nucellus tissue, which results in the megaspores at the micropyle end being unable to develop into a functional megaspore. In Puccinellia tenuiflora, the number of normally developed megaspores is between one and three, and the position of the megaspore that eventually develops into the functional megaspore is not fixed but is usually the first or the second one at the chalazal end, with a higher percentage being the latter one. This finding indicates that some cell degeneration derived from megasporocyte meiosis is genetically determined. 
Predictably, megasporogenesis is correlated with the callose (Yang and Shen, 2003). In Lycium barbarum, callose begins to accumulate on the sidewalls of the megaspores during meiosis. At the dyad stage, except for the cell wall at the chalazal end that did not have callose accumulation, the cell wall at the other locations exhibits a thickened callose wall (Yang et al., 2017). Megasporogenesis in angiosperms has a distinct feature: after the megasporocytes undergo meiosis, only one cell develops into the functional megaspore, and other cells undergo regular degeneration.

The mature embryo sac was formed through mitotic division of the single functional megaspore. The developmental pattern of the embryo sac was the Polygonum type. However, the developmental pattern of the embryo sac in jujube is still controversial. In this study, we found that the developmental pattern of the embryo sac was the Polygonum type, which is consistent with the observation by Srinivasachar (1940). However, Tian and Ma (1987) considered the developmental pattern to be the Allium type. The discrepancy may be attributable to the genetic characteristics in different jujube cultivars, although the specific causes still need to be further investigated.

Dichogamy refers to the maturation of stamens and pistils in one plant or one flower at different times or the male and female sexual functions at different time intervals (Zhao et al., 2011). 'Zhongqiusucui' has hermaphrodite flowers, and the development of its stamen and pistil were asynchronous. Therefore, 'Zhongqiusucui' is dichogamous, which is mainly represented by stamens developing earlier than pistils. When microspore mother cells began meiosis, ovule primordia first appeared in the pistils. In later stages, the development of pistils accelerated, and it was a relatively long period until the two-nucleate embryo sac was formed. When the anther cracked open and started to release pollen, the embryo sac had just matured. Although the development of pistils was later than that of stamens, pollination and fertilization were unaffected because 12 to $16 \mathrm{~h}$ are usually required for pollen to germinate after falling onto the stigma (Wang, 2008). In addition, the germinated pollen requires time to reach the embryo sac. Thus, during this time, the embryo sac has developed to its maturity. The asynchronous development of male and female organs is a mechanism of dichogamy to effectively prolong the pollination period (Gabriela et al., 2008). Similar phenomena have been observed in other plant species, such as Juglans regia (Zhao et al., 2011) and Castanea mollissima.

In 'Zhongqiusucui', the floral bud external morphology was correlated with the development of male and female gametophytes internally. We can understand the development of stamens and pistils by observing the morphological characteristics of floral buds and their sizes, which is helpful for the timely and accurate management of the flowering period, promotes the normal development of pollen and embryo sac, helps in the prediction of flowering time, and assists in artificial pollination. Meanwhile, our study also provided a strong scientific foundation for tissue culture of jujube pollen and embryo sac (Niu et al., 2011; Zhang et al., 2004), polyploid breeding (Xi et al., 2014), and indepth molecular biology studies.

The results from this study showed that although some abnormalities were present during microsporogenesis, and male gametophyte development in 'Zhongqiusucui', the normal development of male gametophyte was unaffected overall. 'Zhongqiusucui' can produce normal mature pollen. There were some abnormal embryo sacs during megasporogenesis and development of female gametophytes. Even if the abnormal embryo sac is temporarily fertilized, there will be obstacles in the subsequent development process. Seeds without endosperm or small embryo seeds, or empty incomplete seeds will be formed. or seeds degenerate and disappear during development. It is speculated that a large number of embryo sac abortion is part of the cause of seed abortion. However, the factors that lead to embryo abortion and a lack of setting fruits may occur during pollination, fertilization, and embryo development. Further studies must be performed to discover the exact causes.

\section{Literature Cited}

Davis, G.L. 1966. Systematic embryology of the angiosperms. John Wiley \& Sons, New York.

Gabriela, G., V. Miguel, G. Jose, and M. Segarra. 2008. Disassortative mating, Sexual specialization, and the evolution of gender dimorphism in heterodichogamous Acer opalus. Evolution 62(7):1676-1688.

He, C.Y. 2009. The anatomical studies on the process of sexual reproduction of Camellia oleifera. Central South University of Forestry and Technology, Changsha (in Chinese)

He, X.Q. and H. Wu. 2013. Mechanisms of developmental programmed cell death in plants Bul. Bot. 48(4):357-370 (in Chinese).

Heslop-Harrison, J. 1966. Cytoplasmic connection between angiosperm meiocytes. Ann. Bot. 30:221-230.

Hu, S.Y. 2005. Angiosperm reproductive biology. Higher Education Press, Beijing (in Chinese).

Huang, F.Y., S.H. Dou, Y.D. Zhang, X.M. Pang, and Y.Y. Li. 2017. Megasporogenesis, microsporogenesis and development of female and male gametophytes of Ziziphus jujuba Mill. Cv. Dongzao. J. Nucl. Agr. Sci. 31(10):19131920 (in Chinese).

Li, H.P. 2009. Plant microscopic technique. Science Press, Beijing (in Chinese).

Liang, C.L., M.J. Liu, and J. Zhao. 2005. Research progress on plant abortion. Mol. Plant Breeding 3(1):117-122 (in Chinese).

Meng, Y., X.H. Wang, and C.H. Wang. 2009. Embryology of Juglans mandshurica Maxim. (I) the microsporogenesis, and the development of male gametophytes. Bull. Bot. Res. 29(06):681684 (in Chinese)

Niu, Y.F., J.Y. Peng, and L. Li. 2011. Morphology of flower organ and anatomical characteristics of microspore different developmental period in Chinese Jujube and Wild Jujube. J. Plant Genet. Resources 12(1):158-162 (in Chinese).

Qu, Z.Z., Y.H. Wang, J.Z. Zhou, S.Q. Peng, X.K. Qi, S.Z. Cheng, and M.J. Liu. 1989. Observation on biological characteristics of date blossom. J. Hebei Agr. Univ. 12(1):1-9 (in Chinese).

Srinivasachar, D. 1940. Embryological studies of some member of Rhanaceae. Proc India Acad B. 11:107-116.

Tian, H.Q. and D.Z. Ma. 1987. The embryological observation on a parthenocarpy's Zizyphus Jujuba Mill. J. Integr. Plant Biol. 29(1):29-33 (in Chinese).

Wang, Q.Y. 1983. The developments of embryo and endosperm of Zizyphus jujuba Mill. J. Integr. Plant Biol. 25(6):526-531 (in Chinese).

Wang, S., X.B. Xie, Q.P. Zhong, Z.Y. Gu, J.Q. Zeng, and J.X. Zeng. 2009. A new Zizyphus jujube cultivar 'Zhongqiusucui'. Acta Hort. Sinica 05:771-781 (in Chinese).

Wang, Y. 2008. Studies on pollination biology of jujube. Hebei Agr. Univ. (in Chinese).

Webbm, C. and B.E.S. Gunning. 1990. Embryo sac development in Arbidopsis thalina. (I). Megasporogenesis, including the microtubular cytoskeleton. Sex. Plant Reprod. 3:244-256.

Whelan, E.D.P. 1974. Discontinuities in the callose wall, inter-meiocyte connections, and cytomixix in angiosperm meiocytes. Can. J. Bot. 53:1219-1224.

Xi, X.J., Q.L. Guo, W.T. Xu, J.F. Zhang, and B.L. Li. 2014. Megasporogenesis, megagametogenesis and induction of $2 \mathrm{n}$ eggs with colchicine in poplar section Aigeiros. Scand. J. For. Res. 29(6):527-536.

Xu, M.T., C.N. He, X.Z. Zhang, J.P. Qu, S.Y. Zhang, and R.T. Xu. 2008. Preparation of hematoxylin dyeing solution and improvement of dyeing method. Chinese J. Clin. Expt. Pathol. 24(3):371-372 (in Chinese).

Yang, C.X. and J.H. Shen. 2003. Megasporogenesis, microsporogenesis and the development of the female and male gametophyte of Puccinellia tenuiflora. J. Wuhan Botanical Res. 6:464470 (in Chinese)

Yang, S.J., L. Peng, H. Bao, and G.L. Mao. 2017. Cytological sectioning study of megasporogensis and female gametophyte (FG) in Lycium barbarum. Acta Botanica Boreali-Occidentalia Sinica 37(8):1533-1539 (in Chinese).

Zeng, M. 1959. Biological characteristics of jujube. Science Press, Beijing (in Chinese).

Zhang, D.D., D. Liu, and X.M. Lv. 2014. The cysteine protease CEP1, a key executor involved in tapetal programmed cell death, regulates pollen development in Arabidopsis. Plant Cell 26(5):2939-2961.

Zhang, H., W.Q. Liang, and D.B. Zhang. 2008 Research progress on tapetum programmed cell death. J. Shanghai Jiaotong Univ. (Agr. Sci.) 26(1):86-90 (in Chinese).

Zhang, X.Y., S.Q. Peng, and Z.H. Guo. 2004. Studies on the pollination, fertilization and embryo development of Chinese jujube (Ziziphus jujuba). Scientia Silvae Sinicae 40(5):210-213 (in Chinese).

Zhao, S.G., H.X. Wang, and Z.H. Zhang. 2011. Research advances on dichogamy of walnut. Hubei Agr. Sci. 50(17):3466-3470, 3475 (in Chinese.

Zou, F., D.Y. Yuan, J.H. Duan, T. Liao, Y.Z. Feng, and C. Gao. 2013. Microsporogenesis and development of male and female ligands of 'Jinsi No. 4' jujube (Zizyphus jujuba). Nonwood Forest Res. 31(2):27-31 (in Chinese). 